\title{
Understanding teacher stress when teaching the developed technology by using electroencephalogram (EEG) signals
}

\author{
Norzaliza Md Nor ${ }^{1 *}$, Sh-Hussain Salleh ${ }^{2}$, Ahmad Zubaidi ${ }^{3}$ \\ ${ }^{1}$ Center for Biomedical Engineering, Universiti Teknologi Malaysia,Johor Baharu, Malaysia \\ ${ }^{2}$ Kulliyyah of Information \& Communication Technology (KICT), Intenational Islamic University Malaysia, Malaysia \\ ${ }^{3}$ Faculty of Medicine, Universiti Sultan Zainal Abidin (UniSZA), Kuala Terengganu, Malaysia
}

\author{
Index Terms \\ Human Stress \\ MFCC \\ MLP \\ Precursor Emotion \\ DASS21
}

Received: 24 July 2016

Accepted: 26 August 2016

Published: 25 October 2016

\begin{abstract}
Life is full of frustration and challenge that could lead to mental stress. Teaching could be one of challenging profession which has become crucial to the society. Teachers uphold such abundance work including teaching, handling students and activities at school. Despite of their overload work, the new developed technology need to be taught by the teacher. The technology is actually consists of critical thinking and hands on activity which requires the teacher to be creative in conveying the knowledge. Thus, an experiment has been conducted to identify the teacher's stress by using electroencephalogram (EEG) signals and Depression Anxiety Stress Scale (DASS21). Affective Space Model (ASM) developed by Russell has been used in this research study which consists of valence and arousal. The study in understanding teacher stress by using EEG and ASM has been found as scarce. The objective of this research study is to identify the stress level of the teacher through their emotion. There are two experiments need to be conducted: first experiment is to profile the subject's basic emotions and second experiment will be answering the Depression Anxiety Stress Scale (DASS21) to induce stress. 10 healthy teachers are recruited for this study. Mel Frequency Cepstral Coefficients (MFCC) is adopted for feature extraction and Multi-Layer Perceptron (MLP) will be used as classifier. The result shows that the emotions appear is more towards negative emotions which depicts stress for the subject after teaching the developed technology. Based on this results, we may do further research on the educational system in Malaysia if it is been embedded with the new technology. Furthermore, the study is beneficial for the early stress detection among teacher.
\end{abstract}

(C) 2016 The Author(s). Published by TAF Publishing.

\section{INTRODUCTION}

Stress happen when our physicals and mental stability has been disturbed with a simple reaction at the stimulus. Stress can also been laid into the teacher due to abandoned works that need to be accomplished. Stress can be appeared as real or perceived, positive or negative which happened whenever our physiological or physical response adapt the changing conditions [1]. Limbic system which referred as emotional brain is the primary area of the brain that relate to stress. Understanding teacher stress after teaching the hands-on activity is found as scarce. Especially when we are trying to analyze it by using Electroencephalogram (EEG) signals. A successful in learning math and science has been derive from hands-on instruction [2] and also reflect to other subjects too. Critical thinking, collaboration, communication and creativity can be fostered by educators through hands-on activity.

Furthermore, it expose the student to explore and discover new things [3]. However, the hands-on activity through developed technology; smart bicycle may lead to stress. It could be negative stress since the teacher may get stressful due to heavy workload that need to be ac-

\footnotetext{
* Corresponding author: Norzaliza Md Nor

${ }^{\dagger}$ Email: norzaliza@iium.edu.my
} 
complished in school. In order, to let the student understand what will they going to learn in hands-on activity, the teacher may need a passion and interest too to wind up the motivational factor among students itself.

In this research study, a technology named smart bicycle has been developed by Center Biomedical Engineering (CBE) is used by the teacher and student to learn cognitive skills through hands-on activity. Teacher is required to teach the student on how to develop a smart bicycle through crocodile clip software as the basic hands-on activity. Crocodile clip is the software that has been used to learn how to make a parallel circuit and serial circuit. Teacher need to introduce the student on the symbol that will be used in making parallel circuit and serial circuit. Students can try several times until they know how to make the circuit by themselves and applied in in developing smart bicycle. In smart bicycle, teacher need to teach the student on how to develop a circuit for horn, lamp, signals and fan that will be attached to the smart bicycle.

Then, we are using EEG signals to identify the level of stress for the teacher. Cognitive process that exposes the human responses to emotional stimuli is actually being captured from the scalp by using EEG signals [4]. The neurons of the brain produce rhythmic signals reflecting the brain activities through several frequency bands. However, detecting EEG signals involves some challenging issues in terms of proper position of the electrodes on scalp and extracting emotion signal instead of multiple signals. In this study, due to the excellent temporal resolution and its portability, the EEG was chosen to capture brain signal in contrast to other devices such Functional magnetic resonance imaging or functional MRI (fMRI) and Positron Emission Tomography (PET).

In order to recognize emotion, the precursor emotion plays a vital role to ensure the exact emotion appears. Precursor emotion is important to identify the emotion that appears before the real emotion exists in people behavior. In precursor emotion, we may know the emotion that influences the people behavior especially in revealing their exact emotion. Thus, in this research study, it investigates the correlation between precursor emotion and stress itself.

\section{A. Understanding Emotion}

In this research study, Russell's model [5] has been adapted to understand human emotion according to the current research [6], [7], [8]. Eight basic emotion, happy, clam, fear, sad, anger, disgust and surprise are commonly accepted according to [9]. In figure 1, Russell's Affective Space Model (ASM) is used to describe emotion through $\mathrm{s}$ two-dimensional approach. Four basic emotions has been selected as basis which derive from a combination of valence and arousal. The brain activation in dynamic approach can be seen by analyzing and understanding the brain waves.

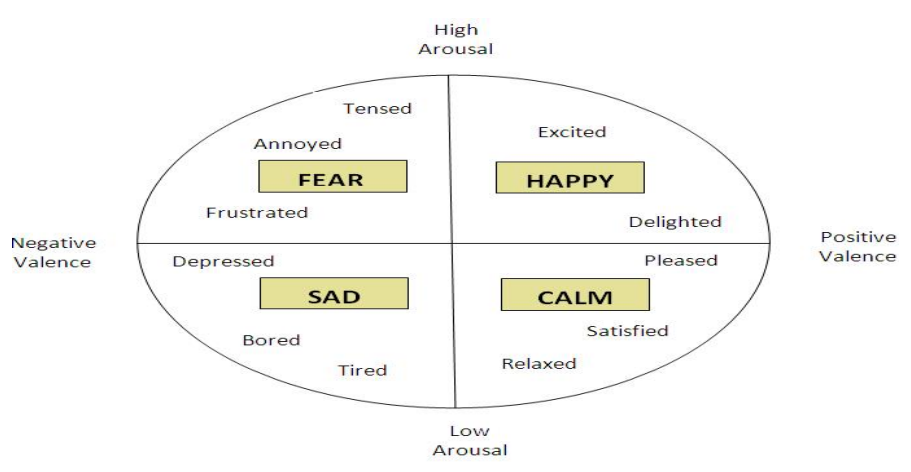

Fig. 1. Russell's Circumplex model of affect

\section{B. Emotion and Eeg}

Electroencephalogram (EEG) device is a diagnostic tool used by the neurologist to record the electrical activity of the brain using numerous electrodes placed on the scalp [10]. The electrical activity produced by the brain cells (neurons activation) and neural circuits can then be captured. These EEG signals can then be used to analyze the student's dynamic emotions and the respective precursor emotion. EEG device has been adopted by many researchers in capturing neural activity in milliseconds from the cortical surface [11] Precursor emotion is something that can form the basis of interpersonal regulation to start [12]. Precursor emotion will be identified by using eyes closed data as it represents the initial emotional state [13].

\section{DASS 21}

Depression, Anxiety, Stress Scales (DASS) is a set of three self-report scales designed to measure the negative emotional states of depression, anxiety and stress. The DASS was constructed not only as a scales to measure conventionally defined emotional states, but to further the process of defining, understanding, and measuring the ubiquitous emotional states usually described as depression, anxiety and stress [14] DASS scales thus meet the needs of both researchers and clinicians who wish to measure current state or change in state over time (e.g., in the course of 
treatment) on the three dimensions of depression, anxiety and stress. Therefore, using DASS in understanding human stress is considered as an important means that needs to be implanted in this study. For this research work, DASS-21 is chosen which contains seven items per scale.

\section{METHODOLOGY}

The flow of the experiment and analysis for the correlation between precursor emotion and subject's stress is shown in figure 2. First, we did the data collection which consists of eyes closed and eyes opened which indicates the resting state of the subject. Then, basic emotion stimuli and then answer the math and science question. After we have the EEG signals(raw data), we sis the pre-processing method which is noise filter by using Ellipord filter. Then, we extract the features by using MFCC with down sampled sampling rate 85 , sampling rate 256,12 : no of cepstral coefficient, 12: no of frames, and $20 \%$ overlapped. Then, we classify the data by using MLP. In MLP, we have two input of data; first data is the randomize combination of four basic emotion (happy, calm, fear and sad) $=[304 \times 176+2]$, including the two target output valence and arousal as the training data. Then, the second input is either the eyes closed data (represent precursor emotion), math questions or science questions as the testing data. We are using two hidden layer and two output (valence and arousal). Output will then being analysed based on combination of positive and negative valence arousal. From this analysis, we can derive the dynamic emotion of the emotion to identify student intrinsic motivational factor in learning math and science.

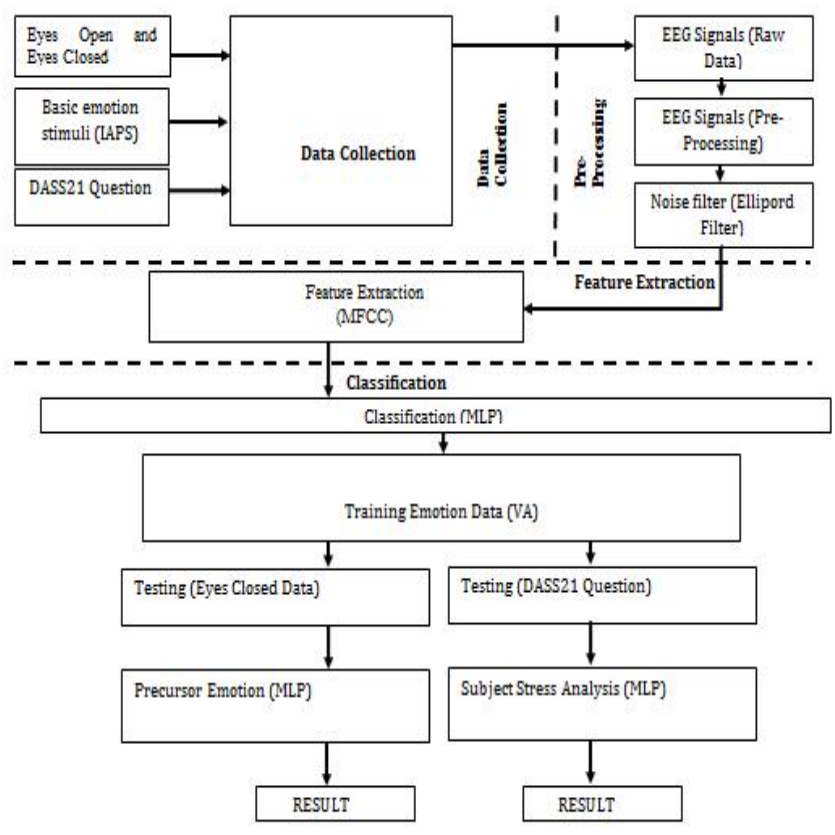

Fig. 2 . Block diagram of experiment

\section{EXPERIMENTAL SETUP}

In this experimental setup, we divided it into two parts. Firstly, the EEG signals of four basic emotions stimuli was collected in order to obtain the ASM. Secondly, the EEG signals of teacher while answering DASS21 questionnaire was also collected. Figure 3 shows the experimental design of the research study. The emotional state of the teacher was derived from the eyes open and eyes closed which shown in figure 2. The teacher need to stare at the blank white screen. A sequence of movie clips will be displayed for one minutes which represents four basic emotions, happy, calm, fear, and sad. Then, the teacher needs to fill up the Self-Assessment Manikin (SAM). Finally, the teacher is required to answer the mathematics and science question. Finally teacher was requested to accomplish a test consisting of DASS21 questionnaire. In this paper, teacher was required to answer 21 questions of depression, anxiety, and stress. 


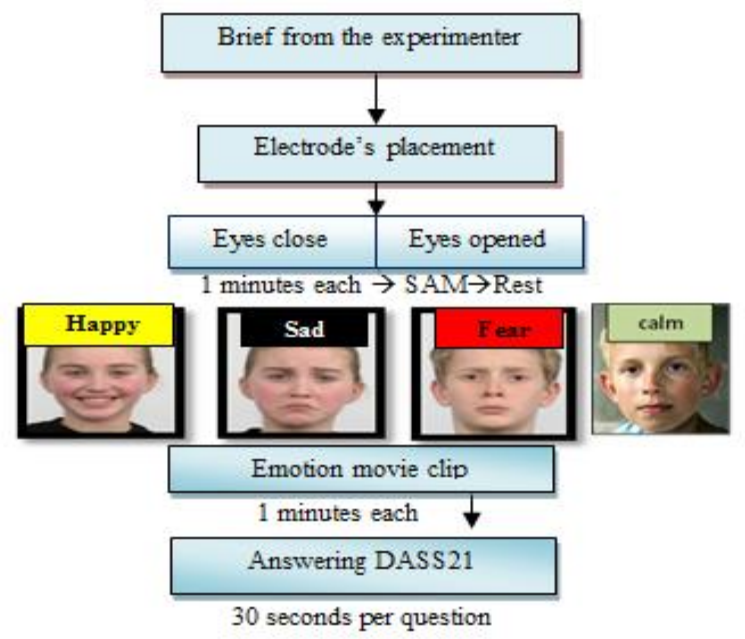

Fig. 3 . Experimental setup and protocol

\section{Stimuli}

The emotion's movie clips were used to obtain emotional responses and one set of mathematics test and science test to identify the student interest. Four basic emotions of happiness, fear, calmness and sadness from the International Affective Picture (IAPS) were used to generate the ASM references of each student. Bernard Bouchard's synthesized musical clips and Gross and Levenson's movie clips were used to elicit emotional response [15] prior to the student doing the mathematics and science tests.

\section{E. Participants}

Ten healthy teachers (five female and five male) were recruited from Sekolah Kebangsaan Taman Universiti 1. Subjects were chosen from the teacher who is involved in the smart learning program in school. Since, we are providing new technology to the teacher and students, thus we tend to identify either the teacher is stressful or not when they have to teach this technology to the students. Since the school project consist of technology that based on Mathematics, Science and English. So the teacher has been selected based on the subject they are teaching.

\section{RESULTS AND OBSERVATION}

In this paper only one subject data were analyzed as a preliminary result. Eyes closed data were first analyzed to identify the precursor emotion. Precursor emotion for subject 1 is shown in figure 4 with combination of calm and sad. Subject seems to have both positive and negative valence also positive and negative arousal while the eyes were closed. In the beginning, subject was showing positive valence and negative arousal which indicates calm emotion. This could mean that the teacher was not sure what to feel and the brain is still having low activation. Then, subject tends to have negative valence and negative arousal which indicates sad emotion. Next, subject showed positive valence and positive arousal which indicates happy emotion. This emotion lasts for 10 seconds. After 10 seconds, subjects tend to have negative valence and positive arousal which indicates fear emotion. These four emotions fluctuate every 10 seconds.

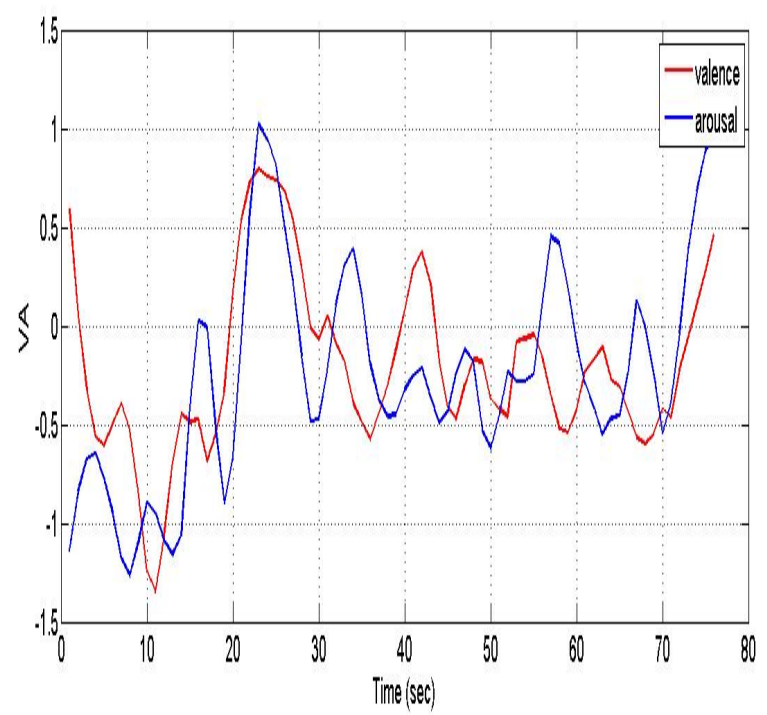

Fig. 4 . Precursor emotion of subject 
TABLE 1

DASS21 SCORING TABLE

\begin{tabular}{llll}
\hline \hline & Depression & Anxiety & Stress \\
\hline Normal & $0-4$ & $0-3$ & $0-7$ \\
Mild & $5-6$ & $4-5$ & $8-9$ \\
Moderate & $7-10$ & $6-7$ & $10-12$ \\
Severe & $11-13$ & $8-9$ & $13-6$ \\
Extremely Severe & $14+$ & $10+$ & $17+(18)$ \\
\hline \hline
\end{tabular}

Question number 1 is "I found it hard to wind down" [16]. According to question number 1 , subject tends to have positive valence and positive arousal which depicts happy emotion. The subject's emotion fluctuates between happy emotions and fear emotion which showing negative arousal and positive valence. This is shown in figure 5.

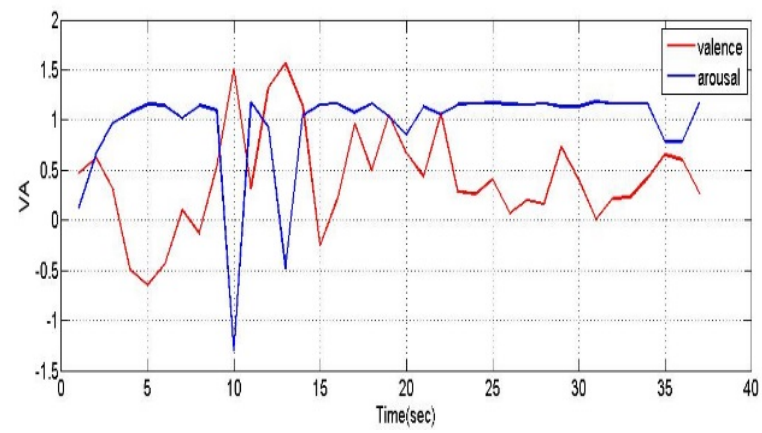

Fig. 5 . Dynamic emotion of subject for question 1

Question number 2 is "I was aware of dryness of my mouth" [16]. For this question, subject tends to have positive valence and positive arousal which depicts happy emotion. The emotion fluctuates between happy and calm emotions which shows positive valence and negative arousal. This is shown in figure 6.

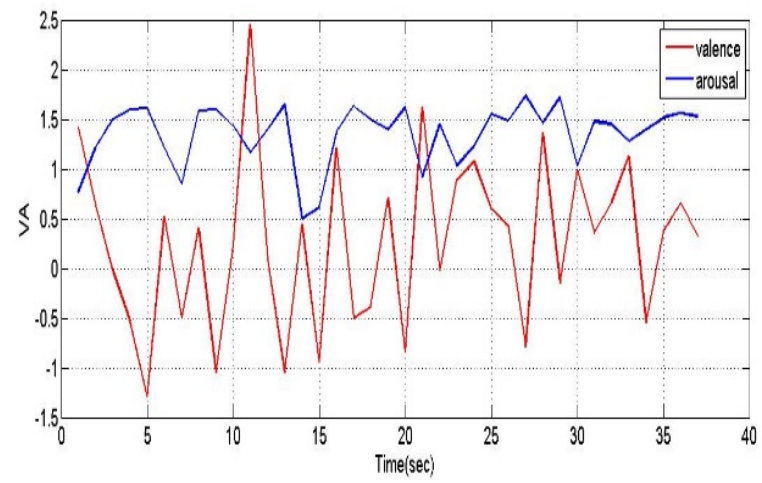

Fig. 6 . Dynamic emotion of subject for question 2 ISSN: $2414-3103$

DOI: $10.20474 /$ japs-2.3.1
Question number 3 is "I couldn't seem to experience any positive feeling at all" [16]. For this question, subject tends to have positive valence and positive arousal which depicts happy emotion. The emotion fluctuates between happy, calm emotion which shows positive valence and negative arousal and fear emotion which shows negative valence and positive arousal. This is shown in figure 7 .

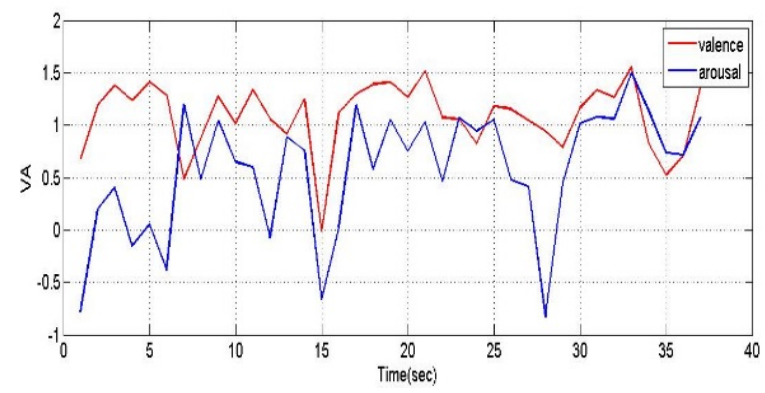

Fig. 7. Dynamic emotion of subject for question 3

Question number 4 is "I experienced breathing difficulty (eg, excessively rapid breathing, breathlessness in the absence of physical exertion)" [16]. For this question, subject tends to have positive valence and positive arousal which depicts happy emotion. The emotion fluctuates between happy, calm emotions which shows positive valence and negative arousal and fear emotion which shows negative valence and positive arousal. This is shown in figure 8 .

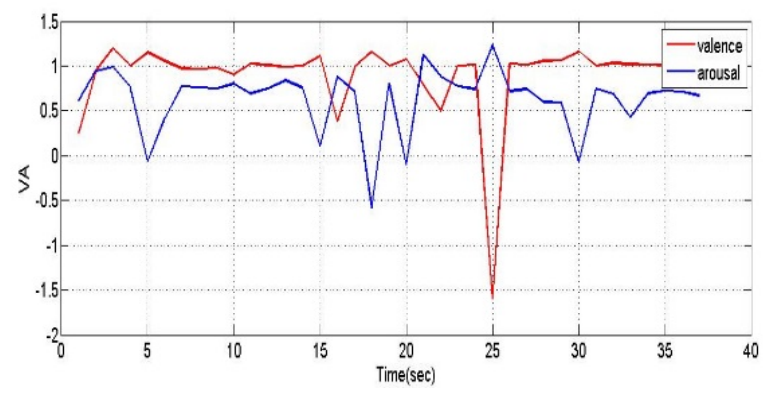

Fig. 8. Dynamic emotion of subject for question 4 TAF 
Question number 5 is "I found it difficult to work up the initiative to do things" [16]. For this question, subject tends to have positive valence and positive arousal which depicts happy emotion. This is shown in figure 9.

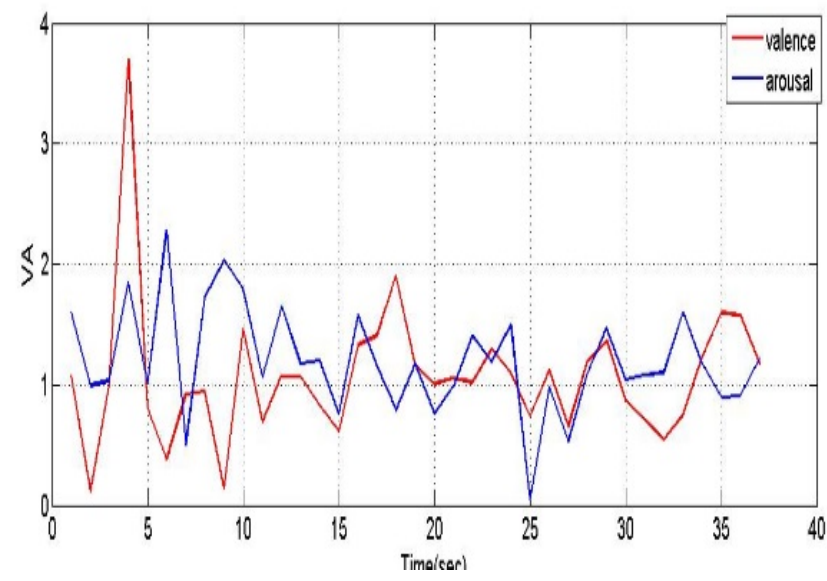

Fig. 9. Dynamic emotion of subject for question 5

Question number 6 is "I tended to over-react to situations" [16]. For this question, subject tends to have positive valence and positive arousal which depicts happy emotion. The emotion fluctuates between happy and calm emotion which shows positive valence and negative arousal. This is shown in figure 10.

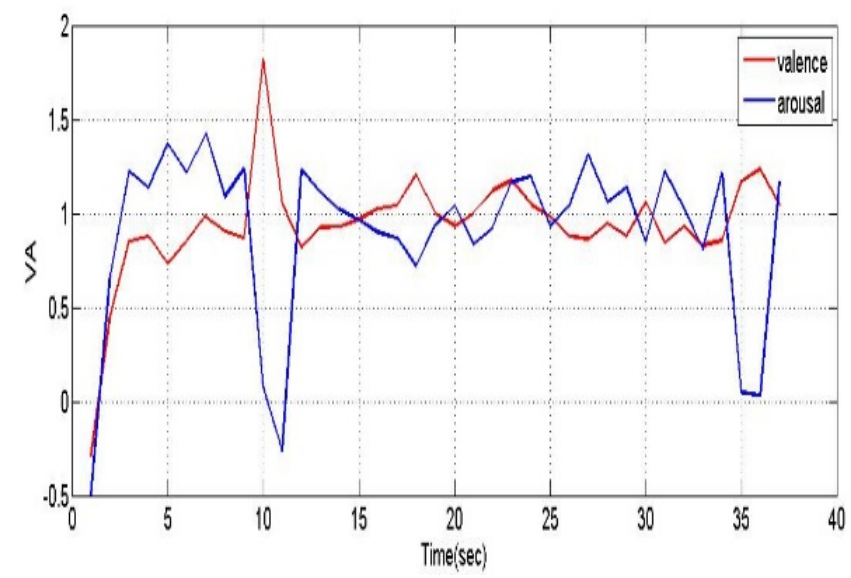

Fig. 10. Dynamic emotion of subject for question 6

Question number 7 is "I experienced trembling (eg, in the hands)" [16]. For this question, subject tends to have positive valence and negative arousal which depicts calm emotion. The emotion fluctuates between calm and sad emotion which shows negative valence and negative arousal. This is shown in figure 11 .

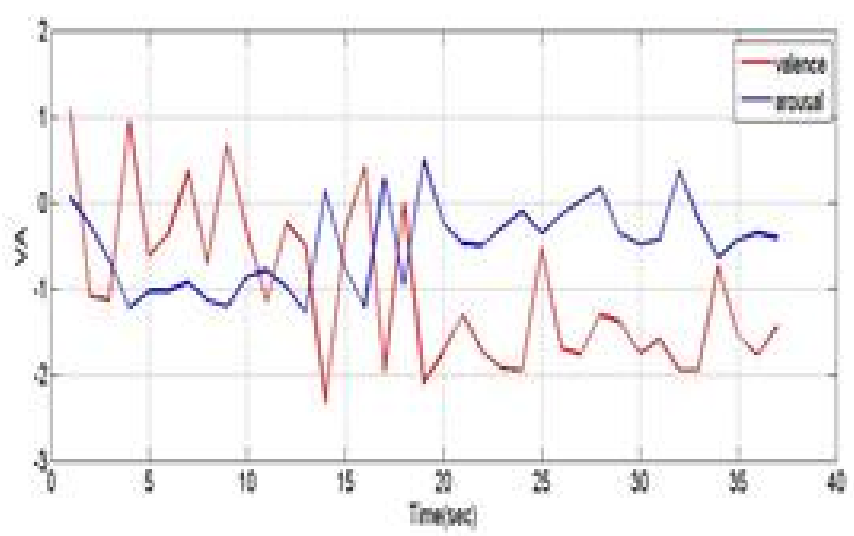

Fig. 11. Dynamic emotion of subject for question 7

Question number 8 is "I felt that I was using a lot of nervous energy" [16]. For this question, subject tends to have negative valence and negative arousal which depicts calm emotion. The emotion fluctuates between sad and calm emotions which shows positive valence and negative arousal. This is shown in figure 12 .

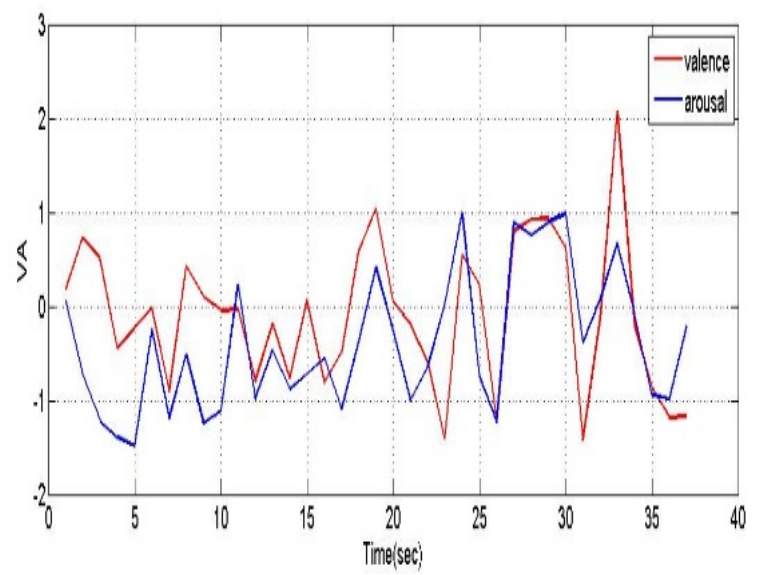

Fig. 12. Dynamic emotion of subject for question 8

Question number 9 is "I was worried about situations in which I might panic and make a fool of myself" [16]. For this question, subject tends to have negative valence and negative arousal which depicts sad emotion. The emotion fluctuates between sad and calm emotions which shows positive valence and negative arousal; and fear emotion towards the end which shows negative valence and positive arousal. This is shown in figure 13 . 


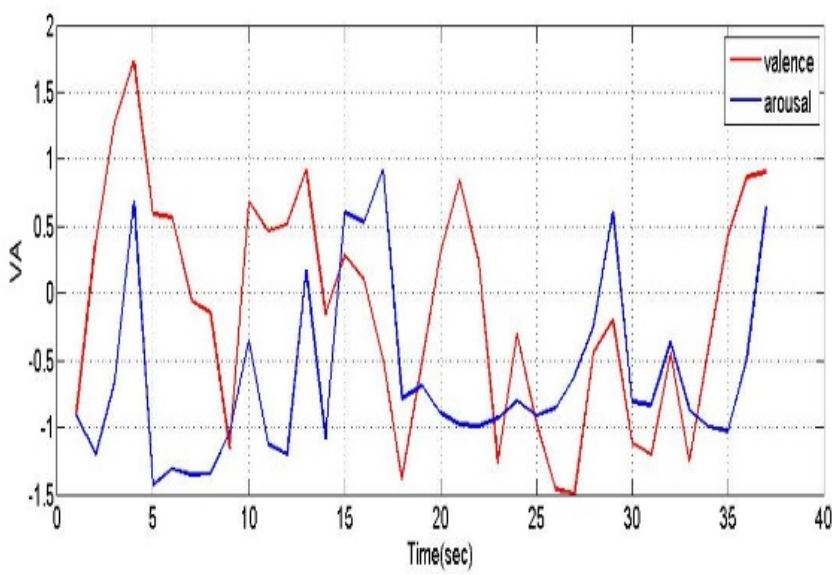

Fig. 13. Dynamic emotion of subject for question 9

Question number 10 is "I felt that I had nothing to look forward to" [16]. For this question, subject tends to have positive valence and negative arousal which depicts calm emotion. The emotion fluctuates between calm and sad emotions which shows negative valence and negative arousal; and fear towards the end which shows negative valence and positive arousal. This is shown in figure 14 .

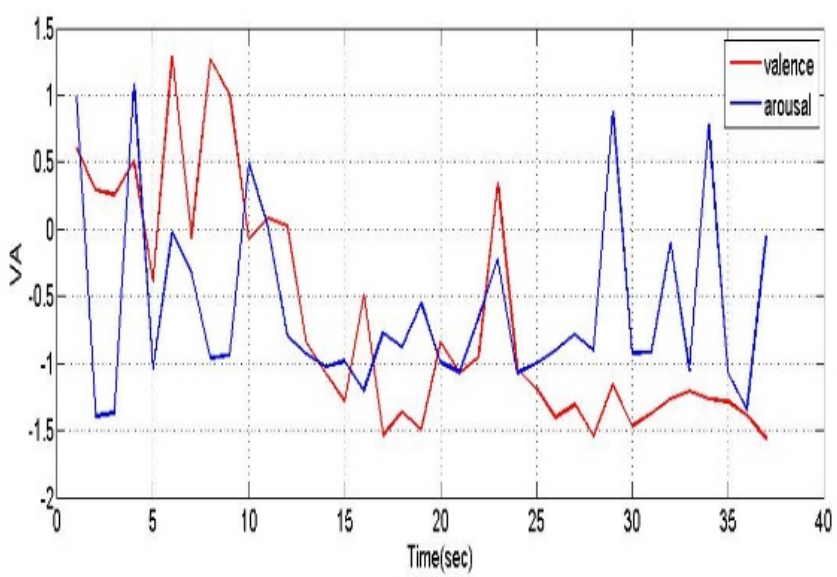

Fig. 14 . Dynamic emotion of subject for question 10

Question number 11 is "I found myself getting agitated" [16]. For this question, subject tends to have positive valence and negative arousal which depicts calm emotion. The emotion fluctuates between calm and sad emotions which shows negative valence and negative arousal, and has fear towards the end which shows negative valence and positive arousal. This is shown in figure 15 .

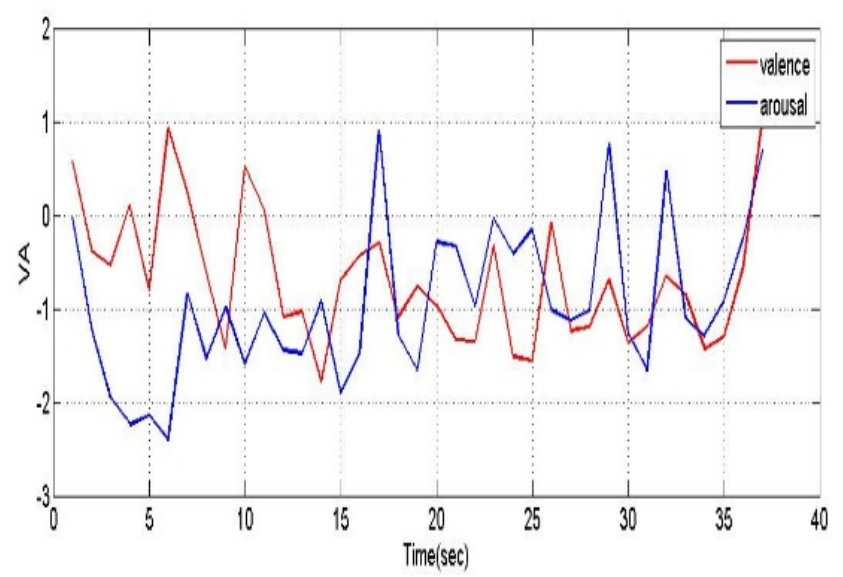

Fig. 15 . Dynamic emotion of subject for question 11

Question number 12 is "I found it difficult to relax" [16]. For this question, subject tends to have positive valence and negative arousal which depicts calm emotion. The emotion fluctuates between calm emotion and sad emotion which shows negative valence and negative arousal; and fear emotion towards the end which shows negative valence and positive arousal. This is shown in figure 16.

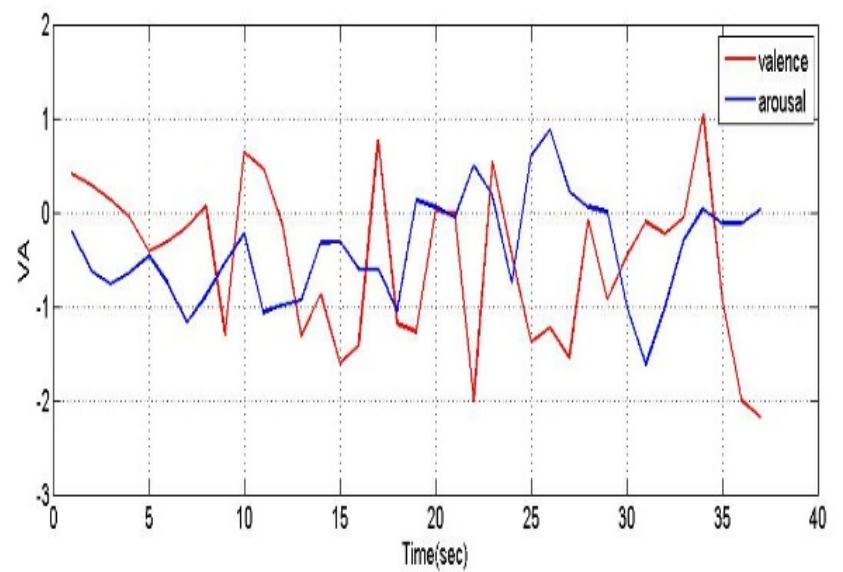

Fig. 16. Dynamic emotion of subject for question 12

Question number 13 is "I felt down-hearted and blue" [16]. For this question, subject tends to have positive valence and positive arousal which depicts happy emotion. The emotion fluctuates between happy and sad emotions which shows negative valence and negative arousal, calm emotion which shows positive valence and negative arousal; and fear emotion towards the end which shows negative valence and positive arousal. This is shown in figure 17. 


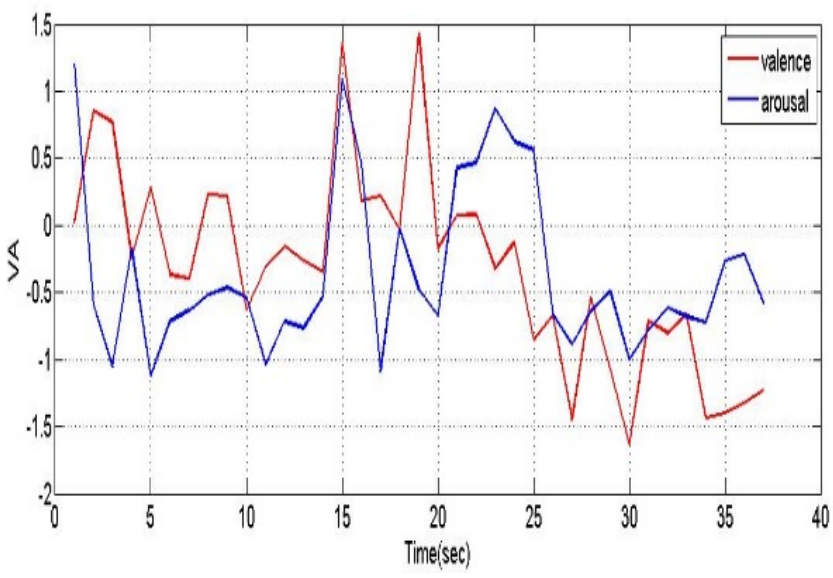

Fig. 17. Dynamic emotion of subject for question 13

Question number 14 is "I was intolerant of anything that kept me from getting on with what I was doing" [16]. For this question, subject tends to have positive valence and positive arousal which depicts happy emotion. The emotion fluctuates between happy and calm emotions which shows positive valence and negative arousal; sad which shows negative valence and negative arousal; and has fear towards the end which shows negative valence and positive arousal. This is shown in figure 18.

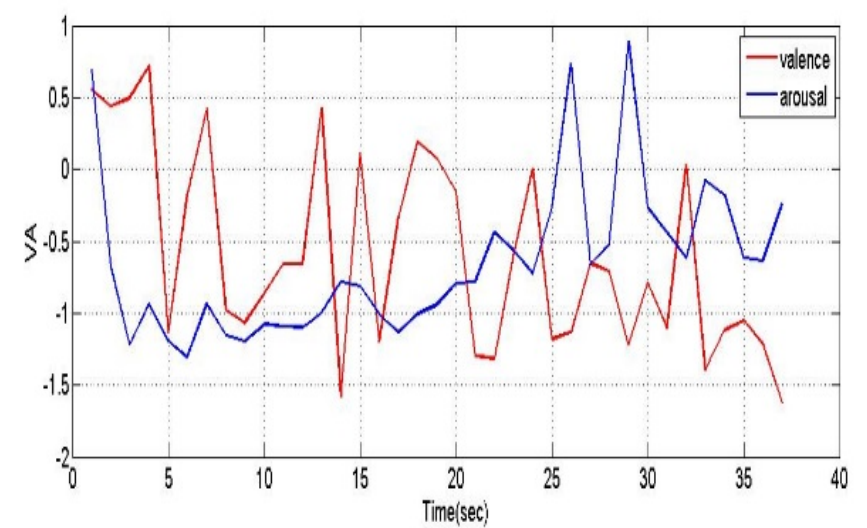

Fig. 18. Dynamic emotion of subject for question 14

Question number 15 is "I felt I was close to panic" [16]. For this question, subject tends to have positive valence and positive arousal which depicts happy emotion. The emotion fluctuates between happy and sad emotions which shows negative valence and negative arousal; and calm emotion towards the end which shows positive valence and negative arousal. This is shown in figure 19.

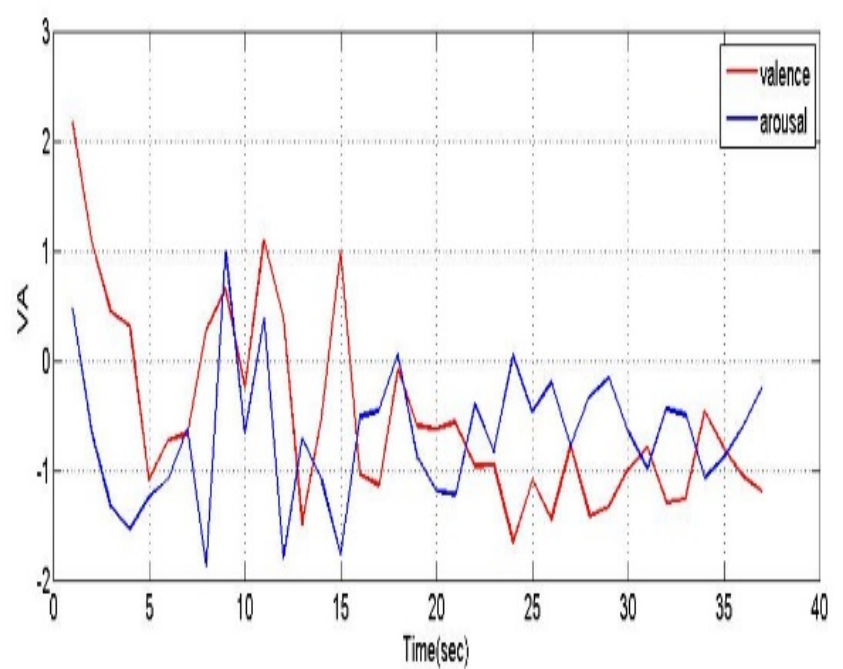

Fig. 19. Dynamic emotion of subject for question 15

Question number 16 is "I was unable to become enthusiastic about anything" [16]. For this question, subject tends to have positive valence and positive arousal which depicts happy emotion. The emotion fluctuates between happy and calm emotions which shows positive valence and negative arousal; and sad emotion towards the end which is showing negative valence and negative arousal. This is shown in figure 20.

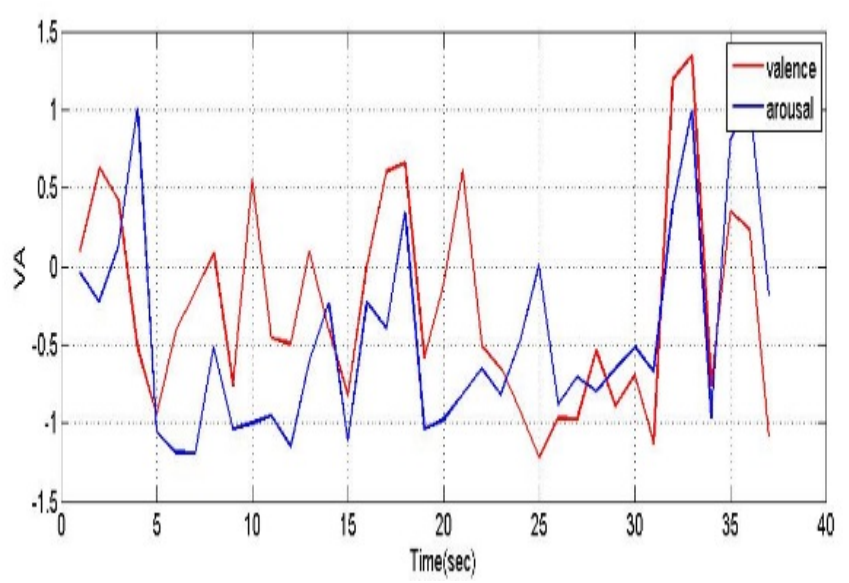

Fig. 20 . Dynamic emotion of subject for question 16

Question number 17 is “I felt I wasn't worth much as a person" [16]. For this question, subject tends to have negative valence and negative arousal which depicts sad emotion. The emotion fluctuates between sad and happy which shows positive valence and positive arousal. This is shown in figure 21. 


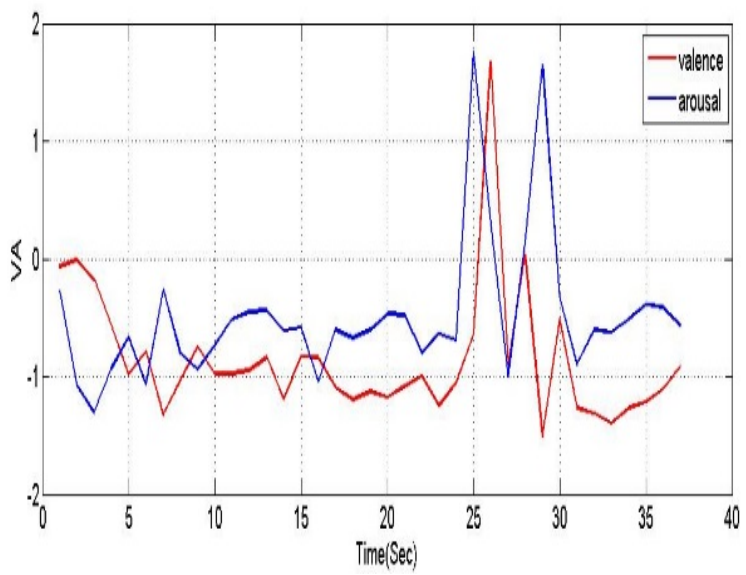

Fig. 21. Dynamic emotion of subject for question 17

Question number 18 is "I felt that I was rather touchy" [16]. For this question, subject tends to have positive valence and positive arousal which depicts happy emotion. The emotion fluctuates between happy and sad emotions which shows negative valence and negative arousal; and fear emotion towards the end which shows negative valence and positive arousal. This is shown in figure 22.

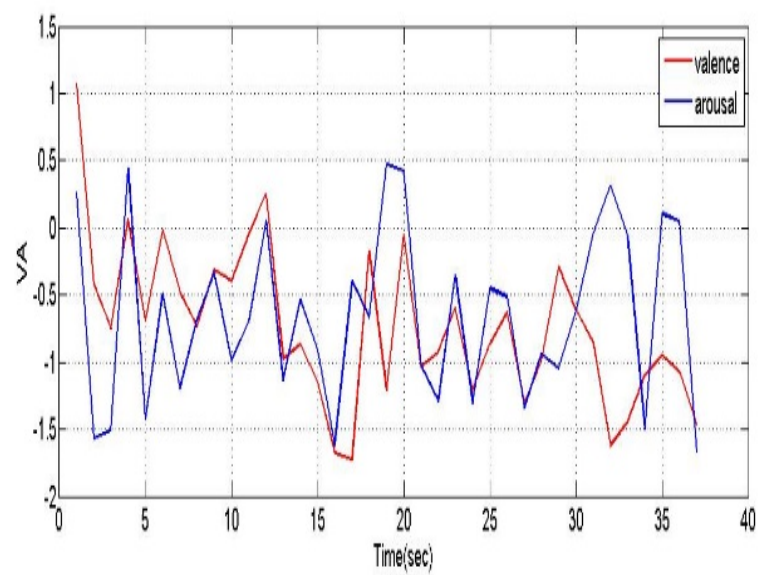

Fig. 22. Dynamic emotion of subject for question 18

Question number 19 is "I was aware of the action of my heart in the absence of physical exertion (eg, sense of heart rate increase, heart missing a beat)" [16]. For this question, subject tends to have positive valence and negative arousal which depicts calm emotion. The emotion fluctuates between calm and sad emotions which shows negative valence and negative arousal. This is shown in figure 23.



Fig. 23. Dynamic emotion of subject for question 19

Question number 20 is "I felt scared without any good reason" [16]. For this question, subject tends to have positive valence and negative arousal which depicts calm emotion. The emotion fluctuates between calm emotion and sad emotion which shows negative valence and negative arousal; and fear emotion towards the end which shows negative valence and positive arousal. This is shown in figure 24.

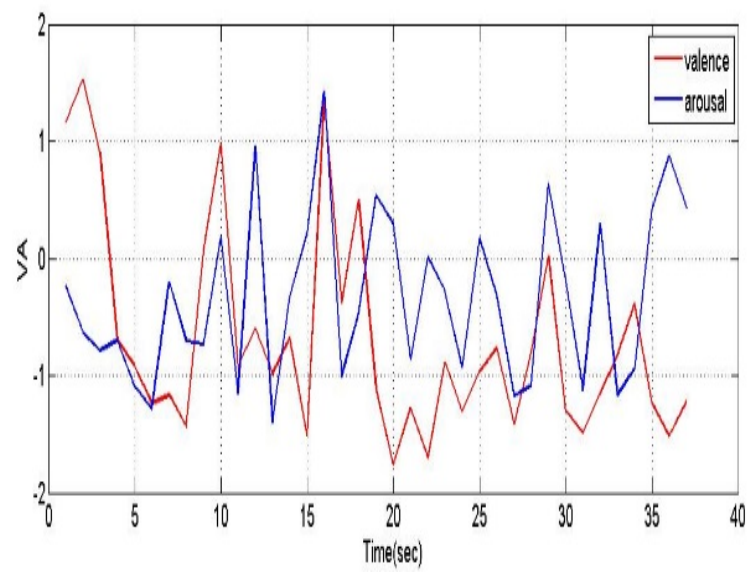

Fig. 24 . Dynamic emotion of subject for question 20

Question number 21 is "I felt that life was meaningless" [16]. For this question, subject tends to have positive valence and negative arousal which depicts calm emotion. The emotion fluctuates between calm and sad emotions which shows negative valence and negative arousal. This is shown in figure 25. 


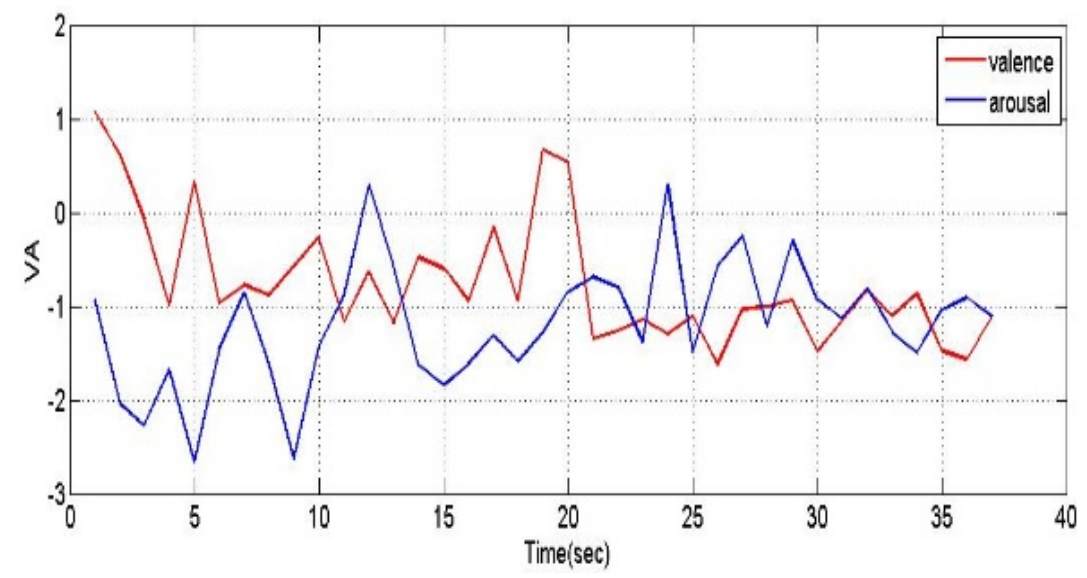

Fig. 25 . Dynamic emotion of subject for question 21

TABLE 2

THE RESULT AFTER APPLYING MULTIPLE BATCH SIZE AND FIXED VALUE FOR DROPOUT $=0.5$

\begin{tabular}{ll}
\hline \hline Question Dynamic Emotion Result & \\
\hline Question 1 & Happy, Fear \\
Question 2 & Happy, Calm \\
Question 3 & Happy, Calm, Fear \\
Question 4 & Happy, Calm, Fear \\
Question 5 & Happy \\
Question 6 & Happy, Calm \\
Question 7 & Calm, Sad \\
Question 8 & Calm, Sad \\
Question 9 & Sad, Calm, Fear \\
Question 10 & Calm, Sad, Fear \\
Question 11 12 & Calm, Sad, Fear \\
Question 12 & Calm, Sad, Fear \\
Question 13 & Happy, Sad, Calm, Fear \\
Question 14 & Happy, Calm, Sad, Fear \\
Question 15 & Happy, Sad, Calm \\
Question 16 & Happy, Calm, Sad \\
Question 17 & Sad, Happy \\
Question 18 & Happy, Sad, Fear \\
Question 19 & Calm, Sad \\
Question 20 & Calm, Sad, Fear \\
Question 21 & Calm, Sad \\
\hline \hline
\end{tabular}

\section{DISCUSSION}

Based on figure 5 until figure 25 and table-2, the first six questions, positive valence and positive arousal which depicts happy emotion appears in all questions. This shows that the subject felt no stress at all. Even though, the sub- ject is having negative valence and positive arousal which depicts fear emotion in question 1 and question 4 but still happy emotions appear in all questions. Perhaps, the first six questions do not induce any stressful thought to the subject. Question 7 and towards the end, the subject is having positive valence and negative arousal which depicts calm 
emotion for the rest of the questions except question number 17 and 18. However, in question 17 and 18, positive valence and positive arousal which depicts happy emotion appears in this question. Thus, this showed that positive valence appears in all questions. However, the negative valence and negative arousal which depicts sad emotion has conquered the subject's emotion starting from question number 7 to 21 even though happy and calm emotion appear to show that he is having positive valence instead of negative valence and negative arousal. This shows that the subject is in a stress condition because $70 \%$ of the emotion appear is sad. The subject might have been stressed due to work abundance in school or the difficulty to handle the new developed technology class. In table 1, it depicts DASS21 score which the subject has scored 18 which fall into extremely severe stress.

According to precursor emotion, all emotion has appeared during eyes closed task and based on DASS21 emotion appearance, it shows that precursor emotion has also influenced the dynamic emotion of the subject. In precursor emotion, subject tends to have all emotions but sad emotion is the most dominant emotion in precursor emotion since it appears three times in almost 30 seconds. Thus, it has influenced the subject's emotion while answering DASS21 questionnaire since sad emotion is the most dominant emotion in this task.

\section{CONCLUSION}

In summary, a dominant negative emotion has been shown in teacher's dynamic emotions while answering the DASS21 questionnaire. This could be due to stress condition that has been occurred during the task. Also note from the results that even though the teacher was having positive emotion for the first six questions but he tends to have sad as dominant emotion from question number 7 towards the end. Precursor emotion plays a vital role when sad emotion appeared throughout the experiment. Consequently, since precursor emotion may exist in the long term memory so that it may influence the human emotion.

According to the results, it shows that it is possible to classify the subject's stress according to precursor emotion and subject's dynamic emotion. Thus, it could help the society especially teachers to trigger stress at the first place and it could also assist the psychologist or psychiatrist to find the best solution to cure the mental stress.

\section{ACKNOWLEDGEMENTS}

Research reported in this publication was supported by vot TRGS:R.J130000.7845.4L840 and Universiti Teknologi Malaysia (UTM).

\section{REFERENCES}

[1] E. S. Elissa B. S. McEwen and J. R. Jeannette, "Embodying psychological thriving: Physical thriving in response to stress," Journal of Social, vol. 54, no. 2, pp. 301-322, 1998. DOI: 10.1111/j.1540-4560.1998.tb01220.x

[2] B. Basista and S. Mathews, "Integrated science and mathematics professional development programs," School Science and Mathematics, vol. 102, no. 7, pp. 359-370, 2002.

[3] B. M. Bass and B. J. Avolio, Improving Organizational Effectiveness through Transformational Leadership. Thousand Oaks, CA: Sage, 1994.

[4] R. F. Farquharson, "The hypothalamus and central levels of autonomic function," The Yale Journal of Biology and Medicine, vol. 12, no. 5, pp. 625, 1942.

[5] J. Russell, "A circumplex model of affect," Journal of Personality and Social Psychology, vol. 39, no. 6, pp. 1161-1178, 1980. DOI: $10.1037 / \mathrm{h} 0077714$

[6] H. Yaacob, I. Karim, A. Wahab and N. Kamaruddin, "Two dimensional affective state distribution of the brain under emotion stimuli," in Annual International Conference of the IEEE Engineering in Medicine and Biology Society, 2012.

DOI: $10.1109 / \mathrm{embc} .2012 .6347374$

[7] H. Yaacob, W. Abdul, I. F. Al Shaikhli and N. Kamaruddin, "CMAC-based computational model of affects (CCMA) for profiling emotion from EEG signals," in The 5th International Conference on Information and Communication Technology for The Muslim World (ICT4M), 2014, pp. 1-6. DOI: 10.1109/ict4m.2014.7020584

[8] M. Othman, H. Yaacob, A. Wahab, I. E. Alshaikli and M. A. Dzulkifli, "A cognitive-affective measurement model based on the 12-point affective circumplex," in 3rd International Conference on Advanced Computer Science Applications and Technologies, 2014. DOI: 10.1109/acsat.2014.8 
[9] P. Ekman, "Basic emotions," in Handbook of Cognition and Emotion, T. Dagleish and M. Power, eds. Sussex, UK: John Wiley \& Sons Ltd, 1999, pp. 45-60.

[10] A. S. Al Mejrad, "Human emotions detection using brain wave signals: A challenging," European Journal of Scientific Research, vol. 44, no. 4, pp. 640-659, 2010.

[11] R. Khosrowabadi, C. Quek, K. K. Ang and A. Wahab, "A biologically inspired feedforward neural network to discriminate emotion from EEG Signal," IEEE Transactions on Neural Networks and Learning Systems, vol. 25, no. 3, pp. 609-620, 2014. DOI: 10.1109 /TNNLS.2013.2280271

[12] L. A. Sroufe, Emotional Development: The Organization of Emotional Life in the Early Years (Cambridge Studies in Social and Emotional Development). Cambridge, UK: Cambridge University Press, 1997, pp. 58-64.

[13] J. Viljaranta, M. K. Lerkkanen, A. M. Poikkeus, K. Aunola and J. E. Nurmi, "Cross-lagged relations between task motivation and performance in arithmetic and literacy in kindergarten," Learning and Instruction, vol. 19, no. 4, pp. 335-344, 2009. DOI: 10.1016/j.learninstruc.2008.06.011

[14] J. R. Crawford and J. D. Henry, "The depression anxiety stress scales (DASS): Normative data and latent structure in a large non-clinical sample," British Journal of Clinical Psychology, vol. 42, no. 2, pp. 111-131, 2003.

DOI: $10.1348 / 014466503321903544$

[15] G. Chanel, J. Kronegg, D. Granjean and T. Pun, "Emotion assessment: Arousal evaluation using EEG's and peripheral physiological signals," in Proceedings International Workshop on Multimedia Content Representation, Classification and Security, Istanbul, Turkey, 2006, pp. 530-537. DOI: 10.1007/11848035_70

[16] S. H. Lovibond and P. F. Lovibond, Manual for the Depression Anxiety Stress Scales. Sydney, Australia: Psychology Foundation, 1995.

— This article does not have any appendix. — 